\title{
On the problem of a thin rigid inclusion embedded in a Maxwell material
}

\section{T. Popova}

Institute of Mathematics and Informatics, North-Eastern Federal University, Yakutsk, Russia

G. Rogerson

School of Computing and Mathematics, Keele University, Staffordshire, ST5 5BG, UK

\begin{abstract}
We consider a plane visco-elastic body, composed of Maxwell material, with a crack and a thin rigid inclusion. The statement of the problem includes boundary conditions in the form of inequalities, together with an integral condition describing the equilibrium conditions of the inclusion. An equivalent variational statement is provided and used to prove the uniqueness of the problem's solution. The analysis is carried out in respect of perfect and non-perfect bonding of the rigid inclusion. Additional smoothness properties of the solutions, namely the existence of the time derivative, are also established.
\end{abstract}

\section{Keywords}

visco-elasticity, crack, rigid inclusion, variational inequality, inequality-type boundary conditions, nonpenetration condition

\section{Introduction}

Obtaining a mathematical description of the equilibrium state of viscoelastic bodies with defects, such as cracks and inclusions, is complicated both by non-stationary equations and non-smooth domains. The classical formulation of problems in domains with cracks assumes boundary conditions in the form of equalities. This scenario has been widely investigated, with the methodology firmly established and properties of the solutions well understood. The approach in our present contribution however, assumes inequality-type conditions on the crack faces; such conditions have a clear physical interpretation and are thus better motivated than their classical counterparts. In this case, the faces of the crack are considered part of the border and restrictions on the displacements in the form of inequalities, and excluding mutual penetration of points of opposite faces, are imposed. In respect of this condition, and since the displacements may have different values for each of the faces, we introduce appropriate jump conditions. Notwithstanding its physically motivated attraction, such a formulation does lead to the certain difficulties. For example, a correct differential formulation requires boundary conditions, in the form of equalities and inequalities, to be prescribed on that part of boundary corresponding to the crack faces. In consequence, this is essentially a problem with unknown boundary conditions. Initially, only non-penetration conditions, in the form of inequalities, are prescribed on the faces of the crack . Based on these conditions, together with further assumptions, we determine the set of admissible displacements for which the solution might be sought. The problem is formulated as a variational inequality and the complete system of differential equations and boundary conditions then derived from this inequality. Additional boundary conditions, derived from a variational inequality, also have a physical interpretation. Further complexity arises because an equivalent variational formulation cannot be established through the minimization of an associated energy functional, as is the case for elastic bodies. The variational formulation is in the form of a so-called variational inequality, allowing analysis of solvability and elucidation of qualitative properties of the solution.

One specific problem involving inequality-type boundary conditions for an inelastic plate, with a crack, is considered in [1]; the analogous equilibrium problem for a $3 \mathrm{D}$ viscoelastic body, of Maxwell type, was 
also investigated in [2]. Additional results, for a wide class of constitutive laws and crack models, were studied in [3], within which the method of variational inequalities proved to be highly effective. We also observe that $[3,4,5,6,7,8,9,10,11]$ include descriptions of these methods within various elastic and viscoelastic applications. Over the last few years, this methodology has been actively used to investigate problems involving inclusions without perfect bonding, results may be found in $[5,12,13,14,15,16]$. A discussion of the problem of a viscoelasticity body with a crack at the boundary of a volume inclusion has also been carried out in [17].

In this present paper, we study the equilibrium problem of a viscoelastic body, of Maxwell type, having a crack and thin rigid inclusion. Existence and uniqueness of the solution are established, with an additional property also obtained relating to the smoothness of solution. This paper is organised as follows. In Section 2 the equilibrium problem is formulated and the appropriate constitute theory briefly reviewed. Within Section 3, the problem of a thin rigid inclusion, with perfect bonding, is considered. A thin rigid inclusion, without perfect bonding, is considered in Section 4. The final problem, that of an inclusion crossing the boundary at zero angle, is considered in Section 5. For this problem, because of the geometric features, and unlike previous cases, Korn's inequality, see [18], cannot be used. Solvability of the problem is established by use of the so-called method of fictitious domains.

\section{Formulation of the problem}

Suppose that in its natural undeformed state, a two-dimensional viscoelastic body $\mathcal{B}$, of Maxwell type, occupies the domain $\Omega \subset R^{2}$ with smooth boundary $\Gamma$. We denote the displacement vector of points in $\mathcal{B}$ by $\mathbf{u}=\left(u_{1}, u_{2}\right)$. The constitutive relation will be assumed in the form

$$
\dot{\sigma}=\mathbf{A} \dot{\varepsilon}+\mathbf{B} \varepsilon
$$

where $\boldsymbol{\varepsilon}$ and $\boldsymbol{\sigma}$ denote components of the infinitesimal strain and stress tensors, respectively, and with a superimposed dot indicating differentiation with respect to time. The components of the strain tensor are related to the displacement gradient through

$$
\varepsilon_{i j}(\mathbf{u})=\frac{1}{2}\left(u_{i, j}+u_{j, i}\right), \quad \mathbf{u}=\mathbf{u}(\mathbf{x}, t), \mathbf{x}=\left(x_{1}, x_{2}\right) \in \Omega, t \in(0, T) .
$$

From the above equations of state, the components of the stress tensor may be represented in the form

$$
\sigma_{i j}(\mathbf{x}, t)=a_{i j k l}(\mathbf{x}) \varepsilon_{k l}(\mathbf{u}(\mathbf{x}, t))+\int_{0}^{t} b_{i j k l}(\mathbf{x}) \varepsilon_{k l}(\mathbf{u}(\mathbf{x}, \tau)) d \tau, i, j=1,2,
$$

where here and henceforth summation over repeated indices is assumed. The coefficients $a_{i j k l}(\mathbf{x}), b_{i j k l}(\mathbf{x}) \in$ $L^{\infty}(\Omega), i, j, k, l=1,2$ are the components of positive definite symmetric tensors $\mathbf{A}$ and $\mathbf{B}$, so for example

$$
\begin{gathered}
a_{i j k l}=a_{j i k l}=a_{k l i j}, \\
a_{i j k l} \xi_{k l} \xi_{i j} \geq c_{0}|\xi|^{2}, \quad \forall \xi_{i j}=\xi_{j i},
\end{gathered}
$$

within which $c_{0}$ is a positive constant and where similar relations hold for $b_{i j k l}$. We emphasise that, in contrast to the elastic case, we cannot calculate the components of stress locally with respect to time; they depend on the full loading history. It is remarked, in passing, that quasi-stationary boundary value problems, for equations using relations of the type presented in (1), were studied in [1], [3], [2], [17].

We now assume that the above mentioned viscoelastic body has a thin rigid inclusion, with shape defined by the curve $\gamma \subset \Omega$; with $\gamma$ assumed smooth, unclosed and without self-intersections. We will denote the unit normal to $\gamma$ by $\boldsymbol{\nu}=\left(\nu_{1}, \nu_{2}\right)$ and, in view of the cut within the domain, assume that $\Omega_{\gamma}=\Omega \backslash \bar{\gamma}$. It is further assumed that we can extend $\gamma$ to the boundary $\Gamma$, so that $\Omega_{\gamma}$ is divided into two sub-domains, $\Omega^{+}$and $\Omega^{-}$, both with Lipschitz boundaries, and with the measure of $\left(\Gamma \cap \partial \Omega^{ \pm}\right)$strictly positive. For notational convenience, it will also assumed that $\gamma^{+}=\partial \Omega^{+} \cap \gamma$ and $\gamma^{-}=\partial \Omega^{-} \cap \gamma$. The direction of the normal $\boldsymbol{\nu}$ is chosen so that if $\boldsymbol{\nu}^{-}$, the external normal to $\partial \Omega^{-}$, coincides with $\boldsymbol{\nu}$, then $\nu^{+}=-\nu$. 
Within the aforementioned framework, we now wish to introduce the concept of a rigid inclusion. To do this, we first introduce the space $R(\gamma)$ of the rigid infinitesimal displacements, see [5], as

$$
R(\gamma)=\left\{\boldsymbol{\rho}=\left(\rho_{1}, \rho_{2}\right) \mid \boldsymbol{\rho}(\mathbf{x})=D \mathbf{x}+\mathbf{G}, \mathbf{x} \in \gamma\right\}
$$

where

$$
D=\left(\begin{array}{cc}
0 & d \\
-d & 0
\end{array}\right), \mathbf{G}=\left(g^{1}, g^{2}\right)
$$

with $d, g^{1}$ and $g^{2}$ constants. We also define another space $R_{\gamma}$ by

$$
R_{\gamma}=\left\{\boldsymbol{\rho}=\left(\rho_{1}, \rho_{2}\right) \mid \boldsymbol{\rho}(\mathbf{x}, t)=D(t) \mathbf{x}+\mathbf{G}(t) \text { on } \gamma \times(0, T)\right\},
$$

where

$$
D(t)=\left(\begin{array}{cr}
0 & d(t) \\
-d(t) & 0
\end{array}\right), \quad \mathbf{G}(t)=\left(g^{1}(t), g^{2}(t)\right), \quad d(t), g^{1}(t), g^{2}(t) \in L^{2}(0, T) .
$$

Note: It is noted that the viscoelastic body $\mathcal{B}$ may be assumed to contain a thin rigid inclusion whenever the function $\mathbf{u}$ on $\gamma \times(0, T)$ coincides with an element of $R_{\gamma}$, that is when

$$
\mathbf{u}=\boldsymbol{\rho}^{0} \text { on } \gamma \times(0, T), \quad \boldsymbol{\rho}^{0} \in R_{\gamma} .
$$

\section{A thin rigid inclusion with perfect bonding}

We now consider the first equilibrium problem, within which our two-dimensional viscoelastic body has a thin rigid inclusion with perfect bonding. Our aim is to find a function $\mathbf{u}$, such that $\mathbf{u}=\boldsymbol{\rho}^{0}$ on $\gamma \times(0, T)$, where $\boldsymbol{\rho}^{0} \in R_{\gamma}$. Moreover, we wish to find a tensor function $\boldsymbol{\sigma}=\left\{\sigma_{i j}\right\}$, satisfying

$$
\begin{gathered}
-\sigma_{i j, j}(\mathbf{x}, t)=f_{i}(\mathbf{x}, t) \quad \text { in } Q_{\gamma}, \quad i=1,2, \\
\sigma_{i j}(\mathbf{x}, t)=a_{i j k l}(\mathbf{x}) \varepsilon_{k l}(\mathbf{u}(\mathbf{x}, t))+\int_{0}^{t} b_{i j k l}(\mathbf{x}) \varepsilon_{k l}(\mathbf{u}(\mathbf{x}, \tau)) d \tau \quad \text { in } Q_{\gamma}, i, j=1,2,
\end{gathered}
$$

where $Q_{\gamma}=\Omega_{\gamma} \times(0, T)$, see Fig.1, and with

$$
\begin{gathered}
\mathbf{u}(\mathbf{x}, t)=\mathbf{0} \quad \text { on } \Gamma \times(0, T), \\
\int_{\gamma}\left[\sigma_{i j}(\mathbf{x}, t) \nu_{j}(\mathbf{x})\right] \bar{\rho}_{i}(\mathbf{x}) d \gamma=0, \quad t \in(0, T), \quad \forall \overline{\boldsymbol{\rho}} \in R(\gamma),
\end{gathered}
$$

with $[\cdot]$ denoting the jump of function on $\gamma$, so for example

$$
[v]=v^{+}-v^{-}
$$

where $v^{+}$and $v^{-}$are the values of $v$ on $\gamma^{+}$and $\gamma^{-}$. We also note that

$$
\begin{gathered}
\sigma_{i j, j}(\mathbf{x}, t)=\left(\frac{\partial \sigma_{1 j}(\mathbf{x}, t)}{\partial x_{j}}, \frac{\partial \sigma_{2 j}(\mathbf{x}, t)}{\partial x_{j}}\right) \\
\sigma_{i j}(\mathbf{x}, t) \nu_{j}(\mathbf{x})=\sigma_{\nu}(\mathbf{x}, t) \nu_{i}(\mathbf{x})+\sigma_{s i}(\mathbf{x}, t), \quad \sigma_{\nu}(\mathbf{x}, t)=\sigma_{i j}(\mathbf{x}, t) \nu_{j}(\mathbf{x}) \nu_{i}(\mathbf{x}) .
\end{gathered}
$$

Within this problem, (2) are the equilibrium equations for a given external force $\mathbf{f}=\left(f_{1}, f_{2}\right)$, (3) describe the viscoelastic state in accordance with the Maxwell model, the boundary condition (4) model the clamping of the body at its boundary and condition (5) is the equilibrium equation of the thin rigid inclusion at time $t$.

To investigate the problem, we begin by considering the function spaces

$$
H_{\gamma}=\left\{\mathbf{v}=\left(v_{1}, v_{2}\right) \in L^{2}\left(0, T ; H^{1}(\Omega)\right) \times L^{2}\left(0, T ; H^{1}(\Omega)\right) \mid \mathbf{v}=\mathbf{0} \text { on } \Gamma \times(0, T),\left.\mathbf{v}\right|_{\gamma \times(0, T)} \in R_{\gamma}\right\}
$$




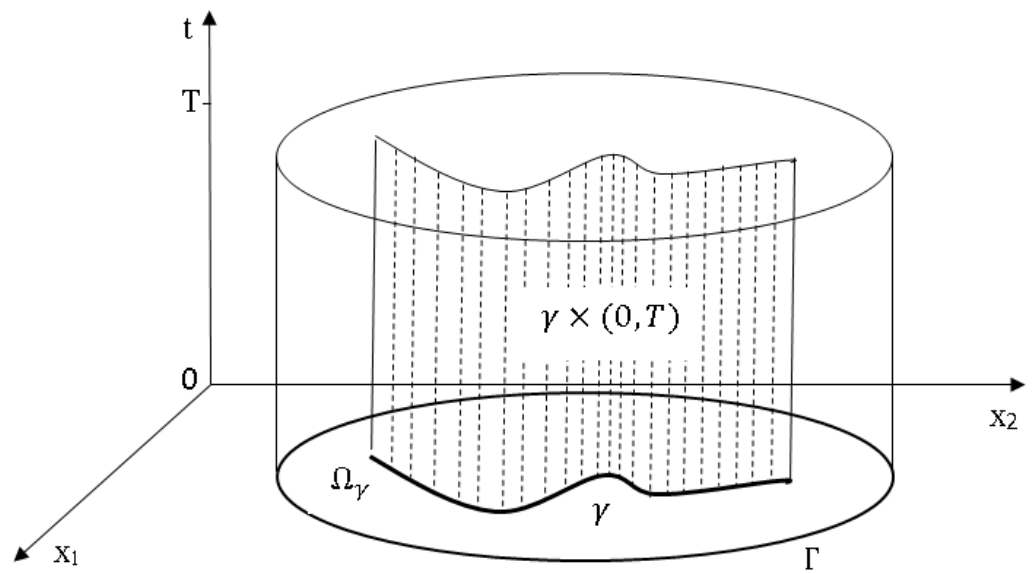

Figure 1: Domain $Q_{\gamma}=\Omega_{\gamma} \times(0, T)$.

and denote by $H_{\gamma}^{*}$ the dual to $H_{\gamma}$. The linear operator $\Lambda: H_{\gamma} \rightarrow H_{\gamma}^{*}$ is now introduced by the formula

$$
\begin{aligned}
(\Lambda \mathbf{u}, \overline{\mathbf{u}})= & \int_{0}^{T}\langle\mathbf{A}(\mathbf{x}) \varepsilon(\mathbf{u}(\mathbf{x}, t)), \varepsilon(\overline{\mathbf{u}}(\mathbf{x}, t))\rangle_{\Omega} d t \\
& +\int_{0}^{T}\left\langle\int_{0}^{t} \mathbf{B}(\mathbf{x}) \varepsilon(\mathbf{u}(\mathbf{x}, \tau)) d \tau, \varepsilon(\overline{\mathbf{u}}(\mathbf{x}, t))\right\rangle_{\Omega} d t, \quad \overline{\mathbf{u}} \in H_{\gamma}
\end{aligned}
$$

with $\langle\cdot, \cdot\rangle_{\Omega}$ a scalar product in $L^{2}(\Omega) ; \varepsilon(\mathbf{u}(\mathbf{x}, t))=\left\{\varepsilon_{i j}(\mathbf{u}(\mathbf{x}, t))\right\} ; \mathbf{A}(\mathbf{x}) \varepsilon(\mathbf{u}(\mathbf{x}, t))=\left\{a_{i j k l}(\mathbf{x}) \varepsilon_{k l}(\mathbf{u}(\mathbf{x}, t))\right\}$; $\mathbf{B}(\mathbf{x}) \boldsymbol{\varepsilon}(\mathbf{u}(\mathbf{x}, \tau))=\left\{b_{i j k l}(\mathbf{x}) \varepsilon_{k l}(\mathbf{u}(\mathbf{x}, \tau))\right\}$.

Definition: We say that an element $\mathbf{u} \in H_{\gamma}$ is a generalized solution of the boundary value problem (2)-(5) with $\mathbf{f} \in H^{1}\left(0, T ; L^{2}(\Omega)\right) \times H^{1}\left(0, T ; L^{2}(\Omega)\right)$ if $\mathbf{u}$ satisfies

$$
\mathbf{u} \in H_{\gamma}, \quad(\Lambda \mathbf{u}, \mathbf{v})=\int_{0}^{T}\langle\mathbf{f}, \mathbf{v}\rangle_{\Omega} d t, \quad \forall \mathbf{v} \in H_{\gamma} .
$$

Theorem 1: There exists a unique solution of the problem (6)

To prove this, we first note Korn's inequality, see [18]

$$
\int_{\Omega} \varepsilon(\mathbf{v}) \varepsilon(\mathbf{v}) d \Omega \geq c_{1}\|\mathbf{v}\|_{\left(H_{0}^{1}(\Omega)\right)^{2}}^{2}, \quad \forall \mathbf{v} \in H_{0}^{1}(\Omega) \times H_{0}^{1}(\Omega),
$$

with the constant $c_{1}$ depending only on the domain $\Omega$. For notational simplicity, we write $\varepsilon(\mathbf{v})$ instead of $\varepsilon_{i j}(\mathbf{v})$ and henceforth suppress the dependence of functions on spatial variables. It is now further deduced that

$$
\begin{aligned}
& (\Lambda \mathbf{u}, \mathbf{u})=\int_{0}^{T}\langle\mathbf{A} \boldsymbol{\varepsilon}(\mathbf{u}(t)), \boldsymbol{\varepsilon}(\mathbf{u}(t))\rangle_{\Omega} d t+\int_{0}^{T}\left\langle\mathbf{B} \boldsymbol{\varepsilon}\left(\int_{0}^{t} \mathbf{u}(\tau) d \tau\right), \boldsymbol{\varepsilon}(\mathbf{u}(t))\right\rangle_{\Omega} d t \\
= & \int_{0}^{T}\langle\mathbf{A} \boldsymbol{\varepsilon}(\mathbf{u}(t)), \boldsymbol{\varepsilon}(\mathbf{u}(t))\rangle_{\Omega} d t+\frac{1}{2}\left\langle\mathbf{B} \boldsymbol{\varepsilon}\left(\int_{0}^{T} \mathbf{u}(t) d t\right), \boldsymbol{\varepsilon}\left(\int_{0}^{T} \mathbf{u}(t) d t\right)\right\rangle_{\Omega} \geq c_{2}\|\mathbf{u}\|_{H_{\gamma}}^{2} .
\end{aligned}
$$


Consequently, it is readily established that

$$
\frac{(\Lambda \mathbf{u}, \mathbf{u})}{\|\mathbf{u}\|_{H_{\gamma}}} \rightarrow+\infty, \quad\|\mathbf{u}\|_{H_{\gamma}} \rightarrow+\infty
$$

i.e., $\Lambda$ is a coercive operator. Since it is monotonic and continuous, we conclude that $\Lambda$ is pseudomonotonic. This implies [10] that a solution to problem (6) exists. Since the operator is strictly monotonic, this solution is necessarily unique. Using the method described in [1], we can also in fact deduce an additional property of the solution of (6), namely the existence of the derivative $\mathbf{u}_{t}(\mathbf{x}, t)$ on $\Omega$. Put $Q=\Omega \times(0, T)$.

Lemma: If $\mathbf{f} \in H^{1}\left(0, T ; L^{2}(\Omega)\right) \times H^{1}\left(0, T ; L^{2}(\Omega)\right)$ then the derivative $\mathbf{u}_{t} \in L^{2}\left(0, T ; H^{1}(\Omega)\right) \times L^{2}\left(0, T ; H^{1}(\Omega)\right)$ of the solution to (6) exists and the following inequality holds

$$
\left\|\mathbf{u}_{t}(t)\right\|_{H_{\gamma}}^{2} \leq c_{3}\left(\left\|\mathbf{f}_{t}(t)\right\|_{\left(L^{2}(Q)\right)^{2}}^{2}+\|\mathbf{f}(t)\|_{\left(L^{2}(Q)\right)^{2}}^{2}\right) .
$$

\section{Proof of Lemma:}

We begin by rearranging (6) as

$$
\begin{array}{r}
\int_{0}^{T}\langle\mathbf{A} \varepsilon(\mathbf{u}(t)), \varepsilon(\mathbf{v}(t))\rangle_{\Omega} d t+\int_{0}^{T}\left\langle\mathbf{B} \boldsymbol{\varepsilon}\left(\int_{0}^{t} \mathbf{u}(\tau) d \tau\right), \boldsymbol{\varepsilon}(\mathbf{v}(t))\right\rangle_{\Omega} d t \\
=\int_{0}^{T}\langle\mathbf{f}(t), \mathbf{v}(t)\rangle_{\Omega} d t, \quad \forall \mathbf{v} \in H_{\gamma},
\end{array}
$$

and let

$$
H_{\gamma}^{1,0}(\Omega)=\left\{\mathbf{v} \in H^{1}(\Omega) \times H^{1}(\Omega) \mid \mathbf{v}=\mathbf{0} \text { on } \Gamma,\left.\mathbf{v}\right|_{\gamma} \in R(\gamma)\right\} .
$$

For $\alpha>0$, consider the function

$$
\mathbf{v}(\theta)=\left\{\begin{array}{rr}
\overline{\mathbf{v}}-\mathbf{u}(t), & \theta \in(t-\alpha, t+\alpha), \\
\mathbf{0}, & \theta \notin(t-\alpha, t+\alpha),
\end{array}\right.
$$

where $\overline{\mathbf{v}} \in H_{\gamma}^{1,0}(\Omega) \times H_{\gamma}^{1,0}(\Omega)$ is a fixed element. Inserting $\mathbf{v}(\theta)$ into (8), and dividing by $2 \alpha$, it may be established that

$$
\begin{gathered}
\frac{1}{2 \alpha} \int_{t-\alpha}^{t+\alpha}\langle\mathbf{A} \varepsilon(\mathbf{u}(t)), \varepsilon(\overline{\mathbf{v}}-\mathbf{u}(t))\rangle_{\Omega} d t+\frac{1}{2 \alpha} \int_{t-\alpha}^{t+\alpha}\left\langle\mathbf{B} \varepsilon\left(\int_{0}^{t} \mathbf{u}(\tau) d \tau\right), \varepsilon(\overline{\mathbf{v}}-\mathbf{u}(t))\right\rangle_{\Omega} d t \\
=\frac{1}{2 \alpha} \int_{t-\alpha}^{t+\alpha}\langle\mathbf{f}(t), \overline{\mathbf{v}}-\mathbf{u}(t)\rangle_{\Omega} d t
\end{gathered}
$$

Hence, as $\alpha \rightarrow 0$, we have for almost all $t \in(0, T)$, see [19], that

$$
\begin{aligned}
\langle\mathbf{A} \varepsilon(\mathbf{u}(t)), \boldsymbol{\varepsilon}(\overline{\mathbf{v}}-\mathbf{u}(t))\rangle_{\Omega}+\left\langle\mathbf{B} \varepsilon\left(\int_{0}^{t} \mathbf{u}(\tau) d \tau\right), \boldsymbol{\varepsilon}(\overline{\mathbf{v}}-\mathbf{u}(t))\right\rangle_{\Omega} \\
=\langle\mathbf{f}(t), \overline{\mathbf{v}}-\mathbf{u}(t)\rangle_{\Omega}, \quad \forall \overline{\mathbf{v}} \in H_{\gamma}^{1,0}(\Omega) \times H_{\gamma}^{1,0}(\Omega) .
\end{aligned}
$$

It is now supposed that $\overline{\mathbf{v}}=\mathbf{u}(t+h)$, and accordingly

$$
\langle\mathbf{A} \varepsilon(\mathbf{u}(t)), \varepsilon(\mathbf{u}(t+h)-\mathbf{u}(t))\rangle_{\Omega}+\left\langle\mathbf{B} \varepsilon\left(\int_{0}^{t} \mathbf{u}(\tau) d \tau\right), \varepsilon(\mathbf{u}(t+h)-\mathbf{u}(t))\right\rangle_{\Omega}
$$




$$
=\langle\mathbf{f}(t), \mathbf{u}(t+h)-\mathbf{u}(t)\rangle_{\Omega} .
$$

We now consider (9) at $t+h$, using $\mathbf{u}(t)$ as a test function for $\overline{\mathbf{v}}$, and consequently deduce that

$$
\begin{gathered}
\langle\mathbf{A} \varepsilon(\mathbf{u}(t+h)), \varepsilon(\mathbf{u}(t)-\mathbf{u}(t+h))\rangle_{\Omega}+\left\langle\mathbf{B} \varepsilon\left(\int_{0}^{t+h} \mathbf{u}(\tau) d \tau\right), \varepsilon(\mathbf{u}(t)-\mathbf{u}(t+h))\right\rangle_{\Omega} \\
=\langle\mathbf{f}(t+h), \mathbf{u}(t)-\mathbf{u}(t+h)\rangle_{\Omega} .
\end{gathered}
$$

We now put

$$
d_{h} \mathbf{v}(t)=\frac{\mathbf{v}(t+h)-\mathbf{v}(t)}{h}, \quad d_{h}^{\tau} \mathbf{v}(t)=\frac{1}{h} \int_{t}^{t+h} \mathbf{v}(\tau) d \tau, \quad h>0
$$

and add (10) and (11) to deduce, in the new notation, that

$$
\left\langle\mathbf{A} \boldsymbol{\varepsilon}\left(d_{h} \mathbf{u}(t)\right), \boldsymbol{\varepsilon}\left(d_{h} \mathbf{u}(t)\right)\right\rangle_{\Omega}=\left\langle d_{h} \mathbf{f}(t), d_{h} \mathbf{u}(t)\right\rangle_{\Omega}-\left\langle\mathbf{B} \boldsymbol{\varepsilon}\left(d_{h}^{\tau} \mathbf{u}(t)\right), \boldsymbol{\varepsilon}\left(d_{h} \mathbf{u}(t)\right)\right\rangle_{\Omega} .
$$

It is now observed that

$$
\left\langle\mathbf{A} \varepsilon\left(d_{h} \mathbf{u}(t)\right), \varepsilon\left(d_{h} \mathbf{u}(t)\right)\right\rangle_{\Omega} \geq c_{4}\left\|d_{h} \mathbf{u}(t)\right\|_{\left(H^{1}(\Omega)\right)^{2}}^{2},
$$

hence (12) implies that

$$
c_{4}\left\|d_{h} \mathbf{u}(t)\right\|_{\left(H^{1}(\Omega)\right)^{2}}^{2} \leq \frac{1}{\lambda}\left\|d_{h} \mathbf{f}(t)\right\|_{\left(L^{2}(\Omega)\right)^{2}}^{2}+\lambda\left\|d_{h} \mathbf{u}(t)\right\|_{\left(L^{2}(\Omega)\right)^{2}}^{2}+\frac{1}{\lambda}\left\|d_{h}^{\tau} \mathbf{u}(t)\right\|_{\left(L^{2}(\Omega)\right)^{2}}^{2}+\lambda\left\|d_{h} \mathbf{u}(t)\right\|_{\left(L^{2}(\Omega)\right)^{2}}^{2} .
$$

We now note that for sufficiently small $\lambda>0$ there exists a constant $c_{5}>0$ such that

$$
\left\|d_{h} \mathbf{u}(t)\right\|_{\left(H^{1}(\Omega)\right)^{2}}^{2} \leq c_{5}\left(\left\|d_{h} \mathbf{f}(t)\right\|_{\left(L^{2}(\Omega)\right)^{2}}^{2}+\left\|d_{h}^{\tau} \mathbf{u}(t)\right\|_{\left(L^{2}(\Omega)\right)^{2}}^{2}\right)
$$

and integrate, for $t$ from 0 to $T-h$, to establish that

$$
\int_{0}^{T-h}\left\|d_{h} \mathbf{u}(t)\right\|_{\left(H^{1}(\Omega)\right)^{2}}^{2} d t \leq c_{5}\left(\int_{0}^{T-h}\left\|d_{h} \mathbf{f}(t)\right\|_{\left(L^{2}(\Omega)\right)^{2}}^{2} d t+\int_{0}^{T-h}\left\|d_{h}^{\tau} \mathbf{u}(t)\right\|_{\left(L^{2}(\Omega)\right)^{2}}^{2} d t\right) .
$$

It is now observed that all smooth functions $v(t)$ satisfy

$$
\int_{0}^{T-h}\left\|d_{h}^{\tau} \mathbf{v}(t)\right\|_{\left(L^{2}(\Omega)\right)^{2}}^{2} d t \leq \int_{0}^{T}\|\mathbf{v}(t)\|_{\left(L^{2}(\Omega)\right)^{2}}^{2} d t,
$$

which when coupled with (14) yields

$$
\int_{0}^{T-h}\left\|d_{h} \mathbf{u}(t)\right\|_{\left(H^{1}(\Omega)\right)^{2}}^{2} d t \leq c_{3}\left(\int_{0}^{T-h}\left\|d_{h} \mathbf{f}(t)\right\|_{\left(L^{2}(\Omega)\right)^{2}}^{2} d t+\int_{0}^{T}\|\mathbf{u}(t)\|_{\left(L^{2}(\Omega)\right)^{2}}^{2} d t\right) .
$$

The first term on the right-hand side of (16) may be rearranged as

$$
\begin{gathered}
\int_{0}^{T-h}\left\|d_{h} \mathbf{f}(t)\right\|_{\left(L^{2}(\Omega)\right)^{2}}^{2} d t=\int_{0}^{T-h}\left\|\frac{\mathbf{f}(t+h)-\mathbf{f}(t)}{h}\right\|_{\left(L^{2}(\Omega)\right)^{2}}^{2} d t \\
=\int_{0}^{T-h}\left\|\frac{1}{h} \int_{t}^{t+h} \mathbf{f}_{\tau}(\tau) d \tau\right\|_{\left(L^{2}(\Omega)\right)^{2}}^{2} d t=\int_{0}^{T-h}\left\|d_{h}^{\tau} \mathbf{f}_{t}(t)\right\|_{\left(L^{2}(\Omega)\right)^{2}}^{2} d t .
\end{gathered}
$$


Since $\mathbf{f}_{t}(t) \in L^{2}(Q) \times L^{2}(Q)$, we can express (15) for $\mathbf{v}=\mathbf{f}_{t}$ as

$$
\int_{0}^{T-h}\left\|d_{h}^{\tau} \mathbf{f}_{t}(t)\right\|_{\left(L^{2}(\Omega)\right)^{2}}^{2} d t \leq \int_{0}^{T}\left\|\mathbf{f}_{t}(t)\right\|_{\left(L^{2}(\Omega)\right)^{2}}^{2} d t
$$

and consequently (16) yields

$$
\int_{0}^{T-h}\left\|d_{h} \mathbf{u}(t)\right\|_{\left(H^{1}(\Omega)\right)^{2}}^{2} d t \leq c_{5}\left(\int_{0}^{T}\left\|\mathbf{f}_{t}(t)\right\|_{\left(L^{2}(\Omega)\right)^{2}}^{2} d t+\int_{0}^{T}\|\mathbf{u}(t)\|_{\left(L^{2}(\Omega)\right)^{2}}^{2} d t\right) .
$$

For a sufficiently small $h_{0}$, but such that $h_{0} \geq h$, it may be shown that

$$
\int_{0}^{T-h_{0}}\left\|d_{h} \mathbf{u}(t)\right\|_{\left(H^{1}(\Omega)\right)^{2}}^{2} d t \leq c_{5}\left(\int_{0}^{T}\left\|\mathbf{f}_{t}(t)\right\|_{\left(L^{2}(\Omega)\right)^{2}}^{2} d t+\int_{0}^{T}\|\mathbf{u}(t)\|_{\left(L^{2}(\Omega)\right)^{2}}^{2} d t\right),
$$

which, passing to the limit as $h \rightarrow 0$, reveals that

$$
\int_{0}^{T-h_{0}}\left\|\mathbf{u}_{t}(t)\right\|_{\left(H^{1}(\Omega)\right)^{2}}^{2} d t \leq c_{5}\left(\int_{0}^{T}\left\|\mathbf{f}_{t}(t)\right\|_{\left(L^{2}(\Omega)\right)^{2}}^{2} d t+\int_{0}^{T}\|\mathbf{u}(t)\|_{\left(H^{1}(\Omega)\right)^{2}}^{2} d t\right) .
$$

Since $h_{0} \geq 0$ is arbitrary, it follows that

$$
\left\|\mathbf{u}_{t}(t)\right\|_{H_{\gamma}}^{2} \leq c_{3}\left(\left\|\mathbf{f}_{t}(t)\right\|_{\left(L^{2}(Q)\right)^{2}}^{2}+\|\mathbf{u}(t)\|_{H_{\gamma}}^{2}\right) .
$$

Thus, the derivative $\mathbf{u}_{t}(x, t)$ exists; moreover taking $\mathbf{v}=\mathbf{u}(t)$ in (8), we obtain

$$
(\Lambda \mathbf{u}, \mathbf{u})=\int_{0}^{T}\langle\mathbf{f}(t), \mathbf{u}(t)\rangle_{\Omega} d t
$$

Taking (7) into account, it may be readily established that

$$
\|\mathbf{u}(t)\|_{H_{\gamma}}^{2} \leq \frac{1}{\lambda}\|\mathbf{f}(t)\|_{\left(L^{2}(Q)\right)^{2}}^{2}+\lambda\|\mathbf{u}(t)\|_{H_{\gamma}}^{2}
$$

or, for small $\lambda>0$, that

$$
\|\mathbf{u}(t)\|_{H_{\gamma}}^{2} \leq c_{6}\|\mathbf{f}(t)\|_{\left(L^{2}(Q)\right)^{2}}^{2} .
$$

Then (18) implies the claim of the lemma. In passing we note that (9) is an equivalent formulation of the problem (6). It is possible to readily confirm that (2)-(5) follow from (9), and conversely, (9) may be derived from (2) to (5).

\section{A thin rigid inclusion without perfect bonding.}

In this section, a rigid inclusion without perfect bonding is considered. To model this, we assume that $\mathcal{B}$ has a crack with shape defined by a curve $\gamma$. The crack has two faces $\gamma^{+}$and $\gamma^{-}$, with a rigidity condition imposed on $\gamma^{-}$. We assume that if the visco-elastic body contains a thin rigid inclusion on one of the crack faces, then the function $\mathbf{u}(\mathbf{x}, t)$ on $\gamma^{-} \times(0, T)$ coincides with an element of $R_{\gamma}$, thus

$$
\mathbf{u}=\boldsymbol{\rho}^{0} \text { on } \gamma^{-} \times(0, T), \quad \boldsymbol{\rho}^{0} \in R_{\gamma} .
$$

Hence, $\mathbf{u}(\mathbf{x}, t)$ will clearly take different values on the two faces of the crack at some current time $t$. A rigid inclusion without perfect bonding means that $\mathbf{u}(\mathbf{x}, t)$ has a jump across $\gamma$. The non-penetration condition,

$$
[\mathbf{u}] \boldsymbol{\nu} \geq 0 \quad \text { on } \gamma \times(0, T),
$$


is also imposed on both sides. This condition precludes the penetration of opposite crack faces into each other. Non-penetration conditions are generally assumed non-linear; for an account of general approaches the reader is referred to [3], [5]. We remark that in the elastic case of thin rigid inclusions without perfect bonding, equilibrium problems have been considered in [13], [14]. These studies elucidate the qualitative properties of solutions as well as discuss the optimal control problem for the crack shape.

A formal statement of the problem is now considered. We wish to find functions $\mathbf{u}=\left(u_{1}, u_{2}\right), \boldsymbol{\rho}^{0} \in R_{\gamma}$, and $\boldsymbol{\sigma}=\left\{\sigma_{i j}\right\}, i, j=1,2$ satisfying:

$$
\begin{gathered}
-\sigma_{i j, j}=f_{i} \quad \text { in } Q_{\gamma}, \quad i=1,2, \\
\boldsymbol{\sigma}(t)=\mathbf{A} \boldsymbol{\varepsilon}(\mathbf{u}(t))+\int_{0}^{t} \mathbf{B} \boldsymbol{\varepsilon}(\mathbf{u}(\tau)) d \tau \quad \text { in } Q_{\gamma} \\
\mathbf{u}=\mathbf{0} \quad \text { on } \Gamma \times(0, T), \\
{[\mathbf{u}] \boldsymbol{\nu} \geq 0, \mathbf{u}^{-}=\boldsymbol{\rho}_{0}, \sigma_{\nu}^{+} \leq 0, \boldsymbol{\sigma}_{s}^{+}=\mathbf{0} \quad \text { on } \gamma \times(0, T),} \\
\sigma_{\nu}^{+}[\mathbf{u}] \boldsymbol{\nu}=0 \quad \text { on } \gamma \times(0, T), \\
\int_{\gamma}[\boldsymbol{\sigma}(t) \boldsymbol{\nu}] \overline{\boldsymbol{\rho}} d \gamma=0, \quad t \in(0, T), \quad \forall \overline{\boldsymbol{\rho}} \in R(\gamma) .
\end{gathered}
$$

We again begin by considering the function spaces

$$
\begin{gathered}
H_{\Gamma}^{1}\left(\Omega_{\gamma}\right)=\left\{\mathbf{v} \in H^{1}\left(\Omega_{\gamma}\right) \times H^{1}\left(\Omega_{\gamma}\right) \mid \mathbf{v}=\mathbf{0} \quad \text { on } \Gamma,\left.\mathbf{v}\right|_{\gamma^{-}} \in R(\gamma)\right\} \\
H_{\gamma^{-}}=\left\{\mathbf{v}=\left(v_{1}, v_{2}\right) \in L^{2}\left(0, T ; H_{\Gamma}^{1}\left(\Omega_{\gamma}\right)\right) \times L^{2}\left(0, T ; H_{\Gamma}^{1}\left(\Omega_{\gamma}\right)\right) \mid \mathbf{v}=\mathbf{0} \text { on } \Gamma \times(0, T),\left.\mathbf{v}\right|_{\gamma^{-} \times(0, T)} \in R_{\gamma}\right\}
\end{gathered}
$$

and the set of admissible displacements

$$
\mathcal{K}_{\gamma}=\left\{\mathbf{v} \in H_{\gamma^{-}} \mid \mathbf{v}(t) \in K_{\gamma}, t \in(0, T)\right\},
$$

where $K_{\gamma}$ is the closed convex subset

$$
K_{\gamma}=\left\{\mathbf{v} \in H_{\Gamma}^{1}\left(\Omega_{\gamma}\right) \mid[\mathbf{v}] \boldsymbol{\nu} \geq 0 \text { on } \gamma\right\}
$$

Definition: We say that an element $\mathbf{u} \in \mathcal{K}_{\gamma}$ is a generalized solution of the boundary value problem (19)-(24), with $\mathbf{f} \in H^{1}\left(0, T ; L^{2}(\Omega)\right) \times H^{1}\left(0, T ; L^{2}(\Omega)\right)$, if $\mathbf{u}$ satisfies the variational inequality

$$
\mathbf{u} \in \mathcal{K}_{\gamma}, \mathbf{u}_{t} \in L^{2}\left(0, T ; H^{1}\left(\Omega_{\gamma}\right)\right) \times L^{2}\left(0, T ; H^{1}\left(\Omega_{\gamma}\right)\right)
$$

$$
\begin{gathered}
\int_{0}^{T}\langle\mathbf{A} \varepsilon(\mathbf{u}(t)), \varepsilon(\overline{\mathbf{v}}(t)-\mathbf{u}(t))\rangle_{\Omega_{\gamma}} d t+\int_{0}^{T}\left\langle\int_{0}^{t} \mathbf{B} \varepsilon(\mathbf{u}(\tau)) d \tau, \varepsilon(\overline{\mathbf{v}}(t)-\mathbf{u}(t))\right\rangle_{\Omega_{\gamma}} d t \\
\geq \int_{0}^{T}\langle\mathbf{f}(t), \overline{\mathbf{v}}(t)-\mathbf{u}(t)\rangle_{\Omega_{\gamma}} d t, \quad \forall \overline{\mathbf{v}} \in \mathcal{K}_{\gamma} .
\end{gathered}
$$

Theorem 2: There exists a unique solution of the problem (25).

Similarly to the previous section, we first establish the existence of a unique solution of the variational inequality (25). Having done so, we verify that this variational inequality is an equivalent statement of (19)-(24), thus establishing unique solvability. Since Korn's inequality is satisfied, it is possible to utilise the established theorem concerning the existence of a solution of the variational inequality for pseudo-monotone operators [10]. This implies that the solution to problem (25) exists. Moreover, since the operator is strictly monotonic, the solution is unique. As in the previously established lemma, we can show that the derivative $\mathbf{u}_{t} \in L^{2}\left(0, T ; H^{1}\left(\Omega_{\gamma}\right)\right) \times L^{2}\left(0, T ; H^{1}\left(\Omega_{\gamma}\right)\right)$ of the solution to (25) exists.

Now we verify that, assuming sufficient smoothness of solutions, (19)-(24) is equivalent to (25). Suppose that (25) has a smooth solution. First, let us deduce from the variational inequality (25) the 
equations and boundary conditions (19)-(24). Analogously to the proof of the previously established Lemma (see the deduction of (9)), we can consider (25) for a fixed $t \in(0, T)$ :

$$
\begin{gathered}
\langle\mathbf{A} \varepsilon(\mathbf{u}(t)), \varepsilon(\overline{\mathbf{v}}-\mathbf{u}(t))\rangle_{\Omega_{\gamma}}+\left\langle\int_{0}^{t} \mathbf{B} \varepsilon(\mathbf{u}(\tau)), \varepsilon(\overline{\mathbf{v}}-\mathbf{u}(t)) d \tau\right\rangle_{\Omega_{\gamma}} \\
\geq\langle\mathbf{f}(t), \overline{\mathbf{v}}-\mathbf{u}(t)\rangle_{\Omega_{\gamma}}, \quad \forall \overline{\mathbf{v}} \in K_{\gamma}, t \in(0, T) .
\end{gathered}
$$

This variational inequality may now be expressed as

$$
\langle\boldsymbol{\sigma}(t), \boldsymbol{\varepsilon}(\overline{\mathbf{v}}-\mathbf{u}(t))\rangle_{\Omega_{\gamma}} \geq\langle\mathbf{f}(t), \overline{\mathbf{v}}-\mathbf{u}(t)\rangle_{\Omega_{\gamma}}, \quad \forall \overline{\mathbf{v}} \in K_{\gamma}, t \in(0, T) .
$$

Here, as previously, we find $\boldsymbol{\sigma}(t)$ using (1), and so it involves integration from 0 to $t$.

Inserting $\overline{\mathbf{v}}=\mathbf{u}(t)+\mathbf{v}, \mathbf{v} \in C_{0}^{\infty}\left(\Omega_{\gamma}\right) \times C_{0}^{\infty}\left(\Omega_{\gamma}\right)$, into (26) and integrating by parts, it is possible to show that (19) holds for $t \in(0, T)$ in $\Omega_{\gamma}$ in the sense of distributions. Using (19), we can now derive the last two conditions of (22). Indeed, take an arbitrary point $x_{0}$ on $\gamma$, denote by $D\left(x_{0}\right)$ a neighborhood of $x_{0}$ and put $D^{+}=\Omega^{+} \cap D\left(x_{0}\right)$. Then take a smooth function $\boldsymbol{\psi}$ with $\operatorname{supp}(\boldsymbol{\psi}) \subset \bar{D}^{+}$, and $\psi_{\nu} \geq 0$ on $\gamma^{+}$, where $\boldsymbol{\psi}=\psi_{\nu} \boldsymbol{\nu}+\boldsymbol{\psi}_{s}$; and upon inserting a test element $\overline{\mathbf{v}}=\mathbf{u}+\boldsymbol{\psi}$ into (25) and integrating by parts, we obtain

$$
\int_{\gamma^{+}} \boldsymbol{\sigma}(t) \boldsymbol{\nu} \boldsymbol{\psi} d \gamma^{+} \leq 0
$$

The vector functions $\boldsymbol{\sigma}(t) \boldsymbol{\nu}$ and $\boldsymbol{\psi}$ may be expressed as

$$
\boldsymbol{\sigma}(t) \boldsymbol{\nu}=\sigma_{\nu}(t) \boldsymbol{\nu}+\boldsymbol{\sigma}_{s}(t), \quad \boldsymbol{\psi}=\psi_{\nu} \boldsymbol{\nu}+\boldsymbol{\psi}_{s} .
$$

Since $\boldsymbol{\psi}$ is arbitrary, it is deduced that $\boldsymbol{\sigma}_{s}(t)=\mathbf{0}$ on $\gamma^{+}$, and accordingly

$$
\int_{\gamma^{+}} \sigma_{\nu}(t) \cdot \psi_{\nu} d \gamma^{+} \leq 0
$$

which by assumptions on $\boldsymbol{\psi}$ implies that $\sigma_{\nu}(t) \leq 0$ on $\gamma^{+}$.

We shall now deduce (24). To do this, take $\widetilde{\mathbf{u}} \in H^{1}(\Omega) \times H^{1}(\Omega)$ with $\widetilde{\mathbf{u}}=\mathbf{0}$ on $\Gamma$ and $\widetilde{\mathbf{u}}=\boldsymbol{\rho}$ on $\gamma^{ \pm}$, where $\boldsymbol{\rho} \in R(\gamma)$, and insert, as a test element, the function $\overline{\mathbf{v}}=\mathbf{u} \pm \widetilde{\mathbf{u}}$ into (26), yielding

$$
\langle\boldsymbol{\sigma}(t), \varepsilon(\widetilde{\mathbf{u}})\rangle_{\Omega_{\gamma}}=\langle\mathbf{f}(t), \widetilde{\mathbf{u}}\rangle_{\Omega_{\gamma}} .
$$

Taking into account the above relations, together with the assumptions on $\widetilde{\mathbf{u}}$, we can write

$$
\int_{\gamma^{+}}[\boldsymbol{\sigma}(t) \boldsymbol{\nu}] \boldsymbol{\rho} d \gamma^{+}=0,
$$

where $\widetilde{\mathbf{u}}=\boldsymbol{\rho}$ on $\gamma, \boldsymbol{\rho} \in R(\gamma)$. Since $\widetilde{\mathbf{u}}$ is an arbitrary function, the last relation necessarily coincides with (24).

Let us now verify the validity of (23). Suppose first that the strict inequality $[\mathbf{u}(t)] \boldsymbol{\nu}>0$ holds at some point $x_{0} \in \gamma$, indicating the absence of contact between the faces of the crack at some time $t$. This inequality must then hold in some neighborhood $D$ of $x_{0}$. Now put $D^{+}=D \cap \Omega^{+}$and choose $\overline{\mathbf{v}}=\mathbf{u}(t) \pm \lambda \boldsymbol{\psi}$, where $\lambda>0$ and $\boldsymbol{\psi}$ is an arbitrary smooth function with $\operatorname{supp}(\boldsymbol{\psi}) \subset \bar{D}^{+}$. Then, for sufficiently small $\lambda$, the element $\overline{\mathbf{v}}$ belongs to $K_{\gamma}$. With the use of (26), we now obtain

$$
\langle\boldsymbol{\sigma}(t), \boldsymbol{\varepsilon}(\boldsymbol{\psi})\rangle_{D^{+}}=\langle\mathbf{f}(t), \boldsymbol{\psi}\rangle_{D^{+}} .
$$

Integrating by parts, and using the relation (19), we infer that

$$
\int_{\gamma \cap \partial D^{+}} \sigma_{\nu}(t) \psi_{\nu} d \gamma=0
$$


This implies that $\sigma_{\nu}^{+}(t)=0$ in a neighborhood of $x_{0}$. It is now supposed that $\sigma_{\nu}^{+}\left(x_{0}, t\right)<0$. In this case, inserting first the test element $\overline{\mathbf{v}}=\mathbf{0}$ and then $\overline{\mathbf{v}}=2 \mathbf{u}(t)$ into (26), we obtain two inequalities, from which it follows

$$
\langle\boldsymbol{\sigma}(t), \boldsymbol{\varepsilon}(\mathbf{u}(t))\rangle_{\Omega_{\gamma}}=\langle\mathbf{f}(t), \mathbf{u}(t)\rangle_{\Omega_{\gamma}} .
$$

Further integration by parts yields

$$
\begin{gathered}
\int_{\gamma^{-}} \boldsymbol{\sigma}(t) \boldsymbol{\nu} \boldsymbol{\rho}^{0}(t) d \gamma^{-}-\int_{\gamma^{+}} \boldsymbol{\sigma}(t) \boldsymbol{\nu} \mathbf{u}(t) d \gamma^{+}=0, \\
\int_{\gamma^{-}} \boldsymbol{\sigma}(t) \boldsymbol{\nu} \boldsymbol{\rho}^{0}(t) d \gamma^{-}-\int_{\gamma^{+}} \boldsymbol{\sigma}(t) \boldsymbol{\nu} \mathbf{u}(t) d \gamma^{+}-\int_{\gamma^{+}} \boldsymbol{\sigma}(t) \boldsymbol{\nu} \boldsymbol{\rho}^{0}(t) d \gamma^{+}+\int_{\gamma^{+}} \boldsymbol{\sigma}(t) \boldsymbol{\nu} \boldsymbol{\rho}^{0}(t) d \gamma^{+}=0,
\end{gathered}
$$

thus

$$
-\int_{\gamma}[\boldsymbol{\sigma}(t) \boldsymbol{\nu}] \boldsymbol{\rho}^{0}(t) d \gamma-\int_{\gamma^{+}} \boldsymbol{\sigma}(t) \boldsymbol{\nu}\left(\mathbf{u}(t)-\boldsymbol{\rho}^{0}(t)\right) d \gamma^{+}=0 .
$$

Taking (23) into account, we may establish that

$$
\int_{\gamma^{+}} \boldsymbol{\sigma}(t) \boldsymbol{\nu}\left(\mathbf{u}(t)-\boldsymbol{\rho}^{0}(t)\right) d \gamma^{+}=0
$$

and so

$$
\int_{\gamma} \sigma_{\nu}^{+}(t) \boldsymbol{\nu}[\mathbf{u}(t)] d \gamma=0
$$

Since the integrand does not change sign along the curve $\gamma$, it follows that

$$
\sigma_{\nu}^{+}(t)[\mathbf{u}(t)] \boldsymbol{\nu}=0
$$

at all points of $\gamma$. Consequently, on assuming that $\sigma_{\nu}^{+}\left(x_{0}, t\right)<0$, we infer that $\left[\mathbf{u}\left(x_{0}, t\right)\right] \boldsymbol{\nu}\left(x_{0}\right)=0$. Thus, for fixed $t$, we have deduced all equations and conditions (19)-(24) from the variational inequality (26). Conversely, multiplying (19) by $\overline{\mathbf{v}}-\mathbf{u}(t)$, with $\overline{\mathbf{v}} \in K_{\gamma}$, and then integrating by parts, we obtain

$$
\langle\boldsymbol{\sigma}(t), \boldsymbol{\varepsilon}(\overline{\mathbf{v}}-\mathbf{u}(t))\rangle_{\Omega_{\gamma}}-\langle\mathbf{f}(t), \overline{\mathbf{v}}-\mathbf{u}(t)\rangle_{\Omega_{\gamma}}=\int_{\gamma^{-}} \boldsymbol{\sigma}(t) \boldsymbol{\nu}(\overline{\mathbf{v}}-\mathbf{u}(t)) d \gamma^{-}-\int_{\gamma^{+}} \boldsymbol{\sigma}(t) \boldsymbol{\nu}(\overline{\mathbf{v}}-\mathbf{u}(t)) d \gamma^{+} .
$$

We now introduce $L$ in the form

$$
L \equiv \int_{\gamma^{-}} \boldsymbol{\sigma}(t) \boldsymbol{\nu}(\overline{\mathbf{v}}-\mathbf{u}(t)) d \gamma^{-}-\int_{\gamma^{+}} \boldsymbol{\sigma}(t) \boldsymbol{\nu}(\overline{\mathbf{v}}-\mathbf{u}(t)) d \gamma^{+},
$$

where it is noted that $L \geq 0$ would imply (26). To establish the non-negativity of $L$, we first note that

$$
\begin{gathered}
L=\int_{\gamma^{-}} \boldsymbol{\sigma}(t) \boldsymbol{\nu}(\overline{\mathbf{v}}-\mathbf{u}(t)) d \gamma^{-}-\int_{\gamma^{+}} \boldsymbol{\sigma}(t) \boldsymbol{\nu}\left(\overline{\mathbf{v}}-\mathbf{u}(t)-\boldsymbol{\rho}-\boldsymbol{\rho}_{0}(t)+\boldsymbol{\rho}+\boldsymbol{\rho}_{0}(t)\right) d \gamma^{+} \\
=\int_{\gamma^{-}} \boldsymbol{\sigma}(t) \boldsymbol{\nu}(\overline{\mathbf{v}}-\mathbf{u}(t)) d \gamma^{-}-\int_{\gamma^{+}} \boldsymbol{\sigma}(t) \boldsymbol{\nu}\left(\boldsymbol{\rho}-\boldsymbol{\rho}_{0}(t)\right) d \gamma^{+}-\int_{\gamma^{+}} \boldsymbol{\sigma}(t) \boldsymbol{\nu}\left(\overline{\mathbf{v}}-\mathbf{u}(t)-\boldsymbol{\rho}+\boldsymbol{\rho}_{0}(t)\right) d \gamma^{+} \\
=-\int_{\gamma}[\boldsymbol{\sigma}(t) \boldsymbol{\nu}]\left(\boldsymbol{\rho}-\boldsymbol{\rho}_{0}(t)\right) d \gamma-\int_{\gamma^{+}} \boldsymbol{\sigma}(t) \boldsymbol{\nu}\left(\overline{\mathbf{v}}-\mathbf{u}(t)-\boldsymbol{\rho}+\boldsymbol{\rho}_{0}(t)\right) d \gamma^{+} \\
=-\int_{\gamma} \sigma_{\nu}^{+}(t) \boldsymbol{\nu}[\overline{\mathbf{v}}] d \gamma+\int_{\gamma} \sigma_{\nu}^{+}(t) \boldsymbol{\nu}[\mathbf{u}(t)] d \gamma \geq 0 .
\end{gathered}
$$

We have therefore shown that the solution to (19)-(24) satisfies the variational inequality (26). Moreover, inequality (25) coincides with (26) for almost all $t \in(0, T)$. Therefore, we have justified the claim that, in view of the equivalence of (19)-(24) to the variational inequality (25), the original problem is uniquely solvable. 


\section{An inclusion crossing the external boundary at zero angle.}

The scenario in which an inclusion $\gamma$ without perfect bonding crosses the boundary $\Gamma$ at a point $\widehat{x}$ with zero angle, implying that the curves $\gamma$ and $\Gamma$ have a common tangent at the point $\widehat{x}$, is now considered. We begin by introducing $\mathbf{f} \in L^{2}\left(0, T ; L^{2}\left(R^{2}\right)\right) \times L^{2}\left(0, T ; L^{2}\left(R^{2}\right)\right)$ and are required to find the functions $\mathbf{u}=\left(u_{1}, u_{2}\right), \boldsymbol{\rho}_{0} \in R_{\gamma}, \boldsymbol{\sigma}=\left\{\sigma_{i j}\right\}, i, j=1,2$, such that

$$
\begin{gathered}
-\sigma_{i j, j}=f_{i} \quad \text { in } Q_{\gamma}, \quad i=1,2 \\
\boldsymbol{\sigma}(t)=\mathbf{A} \boldsymbol{\varepsilon}(\mathbf{u}(t))+\int_{0}^{t} \mathbf{B} \boldsymbol{\varepsilon}(\mathbf{u}(\tau)) d \tau \quad \text { in } Q_{\gamma}, \\
\mathbf{u}=\mathbf{0} \quad \text { on } \Gamma \times(0, T), \\
{[\mathbf{u}] \boldsymbol{\nu} \geq 0, \quad \text { on } \gamma \times(0, T),} \\
\mathbf{u}=\boldsymbol{\rho}_{0} \quad \text { on } \gamma^{-} \times(0, T), \\
\sigma_{\nu} \leq 0, \boldsymbol{\sigma}_{s}=\mathbf{0} \quad \text { on } \gamma^{+} \times(0, T), \\
\left(\sigma_{\nu}\right)^{+}[\mathbf{u}] \boldsymbol{\nu}=0 \quad \text { on } \gamma \times(0, T), \\
\int_{\gamma}[\boldsymbol{\sigma}(t) \boldsymbol{\nu}] \overline{\boldsymbol{\rho}} d \gamma=0, \quad t \in(0, T), \quad \forall \overline{\boldsymbol{\rho}} \in R(\gamma), \quad \overline{\boldsymbol{\rho}}(\widehat{x})=\mathbf{0} .
\end{gathered}
$$

The problem (28)-(35) can be written in terms of the variational inequality

$$
\begin{aligned}
\mathbf{u} \in \mathcal{K}_{\gamma}, \int_{0}^{T}\langle\mathbf{A} \varepsilon(\mathbf{u}(t)), & \boldsymbol{\varepsilon}(\mathbf{v}(t)-\mathbf{u}(t))\rangle_{\Omega_{\gamma}} d t+\int_{0}^{T}\left\langle\mathbf{B} \varepsilon\left(\int_{0}^{t} \mathbf{u}(\tau) d \tau\right), \varepsilon(\mathbf{v}(t)-\mathbf{u}(t))\right\rangle_{\Omega_{\gamma}} d t \\
& \geq \int_{0}^{T}\langle\mathbf{f}(t), \mathbf{v}(t)-\mathbf{u}(t)\rangle_{\Omega_{\gamma}} d t, \quad \forall \mathbf{v} \in \mathcal{K}_{\gamma},
\end{aligned}
$$

with the set $\mathcal{K}_{\gamma}$ as defined in previous sections. In view of the zero angle at the point $\widehat{x}$, we can not use Korn's inequality to obtain the an estimate analogous to (7), see [5], [12] for details. We shall therefore prove solvability in this case by using the fictitious domain method, see for example [4], [14].

Theorem 3: The problem (36) has a unique solution.

To prove this we first add a fictitious domain $\Omega_{0}$, see Fig.2, and introduce the following notation

$$
\Sigma=\Gamma \cap \partial \Omega_{0}, \quad \Sigma^{0}=\Sigma \backslash \partial \Sigma, \quad \Omega^{\gamma}=\Omega_{\gamma} \cup \Omega_{0} \cup \Sigma^{0}, \quad \widetilde{\Gamma}=\left(\partial \Omega^{\gamma}\right) \backslash \gamma^{ \pm} .
$$

We will essentially consider a family of boundary value problems in the extended domain $Q^{\gamma}=\Omega^{\gamma} \times(0, T)$.

For the small parameter $\lambda>0$, we introduce tensors through the relations

$$
\mathbf{A}^{\lambda}=\left\{\begin{array}{cc}
\mathbf{A} & \text { in } \Omega_{\gamma} \\
\lambda^{-1} \mathbf{A} & \text { in } \Omega_{0},
\end{array} \quad \mathbf{B}^{\lambda}=\left\{\begin{array}{cc}
\mathbf{B} & \text { in } \Omega_{\gamma} \\
\lambda^{-1} \mathbf{B} & \text { in } \Omega_{0}
\end{array}\right.\right.
$$

The differential formulation of the equilibrium problem is as follows. We have to find functions $\mathbf{u}^{\lambda}$, $\boldsymbol{\rho}_{0}^{\lambda} \in R_{\gamma}, \boldsymbol{\sigma}^{\lambda}=\left\{\sigma_{i j}^{\lambda}\right\}$, such that

$$
\begin{gathered}
-\sigma_{i j, j}^{\lambda}=f_{i} \quad \text { in } Q^{\gamma}, \quad i=1,2, \\
\boldsymbol{\sigma}^{\lambda}(t)=\mathbf{A}^{\lambda} \varepsilon\left(\mathbf{u}^{\lambda}(t)\right)+\int_{0}^{t} \mathbf{B}^{\lambda} \varepsilon\left(\mathbf{u}^{\lambda}(\tau)\right) d \tau, \text { in } Q^{\gamma},
\end{gathered}
$$




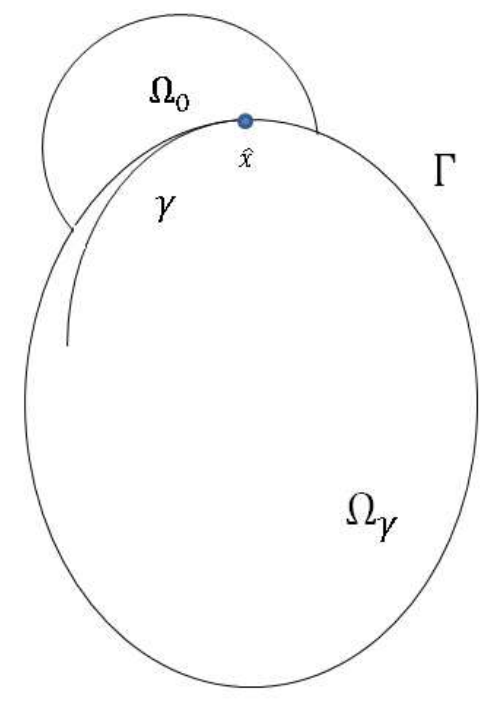

Figure 2: Fictitious domain $\Omega_{0}$ constructing.

$$
\begin{gathered}
\mathbf{u}^{\lambda}=0 \quad \text { on } \widetilde{\Gamma} \times(0, T), \\
{\left[\mathbf{u}^{\lambda}\right] \boldsymbol{\nu} \geq 0, \quad \text { on } \gamma \times(0, T),} \\
\mathbf{u}^{\lambda}=\boldsymbol{\rho}_{0}^{\lambda} \quad \text { on } \gamma^{-} \times(0, T), \\
\sigma_{\nu}^{\lambda} \leq 0, \boldsymbol{\sigma}_{s}^{\lambda}=\mathbf{0} \quad \text { on } \gamma^{+} \times(0, T), \\
\left(\sigma_{\nu}^{\lambda}\right)^{+}\left[\mathbf{u}^{\lambda}\right] \boldsymbol{\nu}=0 \quad \text { on } \gamma \times(0, T), \\
\int_{\gamma}\left[\boldsymbol{\sigma}^{\lambda}(t) \boldsymbol{\nu}\right] \overline{\boldsymbol{\rho}} d \gamma=0, \quad \forall \overline{\boldsymbol{\rho}} \in R(\gamma), t \in(0, T) .
\end{gathered}
$$

To begin, we introduce functional spaces for the problem (37)-(44) in the forms

$$
\begin{gathered}
\widetilde{H}^{1}\left(\Omega^{\gamma}\right)=\left\{\mathbf{v} \in H^{1}\left(\Omega^{\gamma}\right) \times H^{1}\left(\Omega^{\gamma}\right) \mid \mathbf{v}=\mathbf{0} \quad \text { on } \widetilde{\Gamma},\left.\mathbf{v}\right|_{\gamma^{-}} \in R(\gamma)\right\}, \\
H^{\gamma}=\left\{\mathbf{v}=\left(v_{1}, v_{2}\right) \in L^{2}\left(0, T ; \widetilde{H}^{1}\left(\Omega^{\gamma}\right)\right) \times L^{2}\left(0, T ; \widetilde{H}^{1}\left(\Omega^{\gamma}\right)\right) \mid \mathbf{v}=\mathbf{0} \text { on } \widetilde{\Gamma} \times(0, T),\left.\mathbf{v}\right|_{\gamma^{-} \times(0, T)} \in R_{\gamma}\right\},
\end{gathered}
$$

and let $H^{\gamma^{*}}$ denote the dual space to $H^{\gamma}$ and introduce the linear operator $\Lambda^{\lambda}: H^{\gamma} \rightarrow H^{\gamma^{*}}$ through the formula

$$
\left(\Lambda^{\lambda} \mathbf{u}, \overline{\mathbf{u}}\right)=\int_{0}^{T}\left\langle\mathbf{A}^{\lambda} \varepsilon(\mathbf{u}(t)), \varepsilon(\overline{\mathbf{u}}(t))\right\rangle_{\Omega \gamma} d t+\int_{0}^{T}\left\langle\mathbf{B}^{\lambda} \varepsilon\left(\int_{0}^{t} \mathbf{u}(\tau) d \tau\right), \varepsilon(\overline{\mathbf{u}}(t))\right\rangle_{\Omega \gamma} d t, \quad \overline{\mathbf{u}} \in H^{\gamma} .
$$

The set of admissible displacements are defined by

$$
\mathcal{K}^{\gamma}=\left\{\mathbf{v} \in H^{\gamma} \mid \mathbf{v}(t) \in K^{\gamma}, t \in(0, T)\right\},
$$

where

$$
K^{\gamma}=\left\{\mathbf{v} \in \widetilde{H}^{1}\left(\Omega^{\gamma}\right) \mid[\mathbf{v}] \boldsymbol{\nu} \geq 0 \text { on } \gamma\right\}
$$


It is observed that, for each fixed $\lambda$, the problem (37)-(44) is analogous to that posed in (19)-(24). We therefore deduce that the problem (37)-(44) has a unique solution for each fixed $\lambda$ and is equivalent to the variational inequality

$$
\mathbf{u}^{\lambda} \in \mathcal{K}^{\gamma}, \quad\left(\Lambda^{\lambda} \mathbf{u}^{\lambda}, \mathbf{v}-\mathbf{u}^{\lambda}\right) \geq \int_{0}^{T}\left\langle\mathbf{f}, \mathbf{v}-\mathbf{u}^{\lambda}\right\rangle_{\Omega^{\gamma}} d t, \quad \forall \mathbf{v} \in \mathcal{K}^{\gamma} .
$$

This variational inequality can be rewritten in the form

$$
\begin{gathered}
\mathbf{u}^{\lambda} \in \mathcal{K}^{\gamma} \\
\int_{0}^{T}\left\langle\mathbf{A}^{\lambda} \varepsilon\left(\mathbf{u}^{\lambda}(t)\right), \varepsilon\left(\mathbf{v}(t)-\mathbf{u}^{\lambda}(t)\right)\right\rangle_{\Omega^{\gamma}} d t+\int_{0}^{T}\left\langle\mathbf{B}^{\lambda} \varepsilon\left(\int_{0}^{t} \mathbf{u}^{\lambda}(\tau) d \tau\right), \varepsilon\left(\mathbf{v}(t)-\mathbf{u}^{\lambda}(t)\right)\right\rangle_{\Omega \gamma} d t \\
\left.\geq \int_{0}^{T}\left\langle\mathbf{f}(t), \mathbf{v}(t)-\mathbf{u}^{\lambda}(t)\right)\right\rangle_{\Omega \gamma} d t, \quad \forall \mathbf{v} \in \mathcal{K}^{\gamma} .
\end{gathered}
$$

It is now possible to prove that the solution of the original problem (28)-(35) may be obtained as a limit of the solution of (37)-(44). We remark that things are further complicated by the fact that the sets within which (36) or (46) are valid are not the same, $\mathcal{K}_{\gamma}$ for (36) and $\mathcal{K}^{\gamma}$ for (46). To begin, we first insert $\mathbf{v}=\mathbf{0}$ and then $\mathbf{v}=2 \mathbf{u}^{\lambda}$ into (46), yielding two inequalities, from which it follows

$$
\begin{gathered}
\int_{0}^{T}\left\langle\mathbf{A} \varepsilon\left(\mathbf{u}^{\lambda}(t)\right), \boldsymbol{\varepsilon}\left(\mathbf{u}^{\lambda}(t)\right)\right\rangle_{\Omega_{\gamma}} d t+\int_{0}^{T}\left\langle\mathbf{B} \varepsilon\left(\int_{0}^{t} \mathbf{u}^{\lambda}(\tau) d \tau\right), \boldsymbol{\varepsilon}\left(\mathbf{u}^{\lambda}(t)\right)\right\rangle_{\Omega_{\gamma}} d t \\
+\frac{1}{\lambda} \int_{0}^{T}\left\langle\mathbf{A} \varepsilon\left(\mathbf{u}^{\lambda}(t)\right), \boldsymbol{\varepsilon}\left(\mathbf{u}^{\lambda}(t)\right)\right\rangle_{\Omega_{0}} d t+\frac{1}{\lambda} \int_{0}^{T}\left\langle\mathbf{B} \varepsilon\left(\int_{0}^{t} \mathbf{u}^{\lambda}(\tau) d \tau\right), \varepsilon\left(\mathbf{u}^{\lambda}(t)\right)\right\rangle_{\Omega_{0}} d t \\
=\int_{0}^{T}\left\langle\mathbf{f}(t), \mathbf{u}^{\lambda}(t)\right\rangle_{\Omega_{\gamma}} d t .
\end{gathered}
$$

We now let $\lambda \in\left(0, \lambda_{0}\right)$, where $\lambda_{0}$ is fixed. Then from positive definiteness properties of the tensors $\mathbf{A}$ and $\mathbf{B}$, together with the above equation, we derive the uniform (in $\lambda$ ) estimates

$$
\begin{gathered}
\left\|\mathbf{u}^{\lambda}(t)\right\|_{\left(L^{2}\left(0, T ; H^{1}\left(\Omega^{\gamma}\right)\right)\right)^{2}} \leq c_{7}, \quad\left\|\mathbf{u}^{\lambda}(t)\right\|_{\left(L^{2}\left(0, T ; H^{1}\left(\Omega_{0}\right)\right)\right)^{2}} \leq c_{8} \lambda, \\
\left\|\int_{0}^{t} \mathbf{u}^{\lambda}(\tau) d \tau\right\|_{\left(L^{2}\left(0, T ; \widetilde{H}^{1}\left(\Omega^{\gamma}\right)\right)\right)^{2}} \leq c_{9} .
\end{gathered}
$$

Choosing a subsequence, if necessary, we assume that as $\lambda \rightarrow 0$

$$
\begin{aligned}
\mathbf{u}^{\lambda} & \rightarrow \mathbf{u}^{0} \quad \text { weakly in }\left(L^{2}\left(0, T ; \widetilde{H}^{1}\left(\Omega^{\gamma}\right)\right)\right)^{2}, \\
\mathbf{u}^{\lambda} & \rightarrow \mathbf{0} \quad \text { strongly in }\left(L^{2}\left(0, T ; H^{1}\left(\Omega_{0}\right)\right)\right)^{2}, \\
\int_{0}^{t} \mathbf{u}^{\lambda}(\tau) d \tau & \rightarrow \int_{0}^{t} \mathbf{u}^{0}(\tau) d \tau \quad \text { weakly in }\left(L^{2}\left(0, T ; \widetilde{H}^{1}\left(\Omega^{\gamma}\right)\right)\right)^{2} .
\end{aligned}
$$

Now we take an arbitrary function $\mathbf{v} \in \mathcal{K}_{\gamma}$ and extend it to $\Omega_{0} \times(0, T)$, in which it takes the value zero. Keeping to extended function notation, we can conclude that $\mathbf{v} \in \mathcal{K}^{\gamma}$. The extended function $\mathbf{v}$ is now inserted into (46), yielding

$$
\int_{0}^{T}\left\langle\mathbf{A} \varepsilon\left(\mathbf{u}^{\lambda}(t)\right), \boldsymbol{\varepsilon}\left(\mathbf{v}(t)-\mathbf{u}^{\lambda}(t)\right)\right\rangle_{\Omega_{\gamma}} d t+\int_{0}^{T}\left\langle\mathbf{B} \varepsilon\left(\int_{0}^{t} \mathbf{u}^{\lambda}(\tau) d \tau\right), \boldsymbol{\varepsilon}\left(\mathbf{v}(t)-\mathbf{u}^{\lambda}(t)\right)\right\rangle_{\Omega_{\gamma}} d t
$$




$$
\begin{gathered}
\geq \frac{1}{\lambda} \int_{0}^{T}\left\langle\mathbf{A} \boldsymbol{\varepsilon}\left(\mathbf{u}^{\lambda}(t)\right), \boldsymbol{\varepsilon}\left(\mathbf{u}^{\lambda}(t)\right)\right\rangle_{\Omega_{0}} d t+\frac{1}{\lambda} \int_{0}^{T}\left\langle\mathbf{B} \boldsymbol{\varepsilon}\left(\int_{0}^{t} \mathbf{u}^{\lambda}(\tau) d \tau\right), \boldsymbol{\varepsilon}\left(\mathbf{u}^{\lambda}(t)\right)\right\rangle_{\Omega_{0}} d t+ \\
+\int_{0}^{T}\left\langle\mathbf{f}(t), \mathbf{v}(t)-\mathbf{u}^{\lambda}(t)\right\rangle_{\Omega_{\gamma}} d t-\int_{0}^{T}\left\langle\mathbf{f}(t), \mathbf{u}^{\lambda}(t)\right\rangle_{\Omega_{0}} d t .
\end{gathered}
$$

Taking in account that

$$
\liminf \left(\frac{1}{\lambda} \int_{0}^{T}\left\langle\mathbf{A} \varepsilon\left(\mathbf{u}^{\lambda}(t)\right), \boldsymbol{\varepsilon}\left(\mathbf{u}^{\lambda}(t)\right)\right\rangle_{\Omega_{0}}+\frac{1}{\lambda} \int_{0}^{T}\left\langle\mathbf{B} \varepsilon\left(\int_{0}^{t} \mathbf{u}^{\lambda}(\tau) d \tau\right), \varepsilon\left(\mathbf{u}^{\lambda}(t)\right)\right\rangle_{\Omega_{0}}\right) \geq 0,
$$

we pass to the limit in (47) and have

$$
\begin{gathered}
\int_{0}^{T}\left\langle\mathbf{A} \boldsymbol{\varepsilon}\left(\mathbf{u}^{0}(t)\right), \boldsymbol{\varepsilon}\left(\mathbf{v}(t)-\mathbf{u}^{0}(t)\right)\right\rangle_{\Omega_{\gamma}} d t+\int_{0}^{T}\left\langle\mathbf{B} \boldsymbol{\varepsilon}\left(\int_{0}^{t} \mathbf{u}^{0}(\tau) d \tau\right), \boldsymbol{\varepsilon}\left(\mathbf{v}(t)-\mathbf{u}^{0}(t)\right)\right\rangle_{\Omega_{\gamma}} d t \geq \\
\geq \int_{0}^{T}\left\langle\mathbf{f}(t), \mathbf{v}(t)-\mathbf{u}^{0}(t)\right\rangle_{\Omega_{\gamma}} d t .
\end{gathered}
$$

Since $\mathbf{u}^{\lambda} \in \mathcal{K}^{\gamma}$, it is then the case that $\mathbf{u}^{0} \in \mathcal{K}^{\gamma}$. The restriction of $\mathbf{u}^{0}$ on $Q_{\gamma}$ belonging to $\mathcal{K}_{\gamma}$, together with inequality (48), are fulfilled for all $\mathbf{v} \in \mathcal{K}_{\gamma}$. Hence, we can write (48) in the form

$$
\begin{gathered}
\mathbf{u} \in \mathcal{K}_{\gamma}, \quad \int_{0}^{T}\langle\mathbf{A} \varepsilon(\mathbf{u}(t)), \boldsymbol{\varepsilon}(\mathbf{v}(t)-\mathbf{u}(t))\rangle_{\Omega_{\gamma}} d t+\int_{0}^{T}\left\langle\mathbf{B} \varepsilon\left(\int_{0}^{t} \mathbf{u}(\tau) d \tau\right), \boldsymbol{\varepsilon}(\mathbf{v}(t)-\mathbf{u}(t))\right\rangle_{\Omega_{\gamma}} d t \\
\geq \int_{0}^{T}\langle\mathbf{f}(t), \mathbf{v}(t)-\mathbf{u}(t)\rangle_{\Omega_{\gamma}} d t, \quad \forall \mathbf{v} \in \mathcal{K}_{\gamma} .
\end{gathered}
$$

The last inequality coincides with (36). Thus, the restriction of $\mathbf{u}^{0}$ on $Q_{\gamma}$ coincides with the solution $\mathbf{u}$ of (36). So, the solvability of (36) is therefore confirmed and established as unique, proof of the Theorem then being complete. In conclusion we remark that in the case of zero angle between $\gamma$ and $\Gamma$ we can not obtain the existence of the derivative $\mathbf{u}_{t}$ of the solution to (36) because we do not have the Korn's inequality in the domain $\Omega_{\gamma}$.

\section{Acknowledgement}

This work was largely completed whilst GAR was visiting the North-Eastern Federal University. Generous local financial support and kind hospitality is very gratefully acknowledged.

\section{References}

1. Khludnev AM. On equilibrium problem for a plate having a crack under the creep condition. Control and Cybernetics. 1996; 25 (5): 1015-1030.

2. Popova TS. The equilibrium problem for a linear viscoelastic body with a crack. Mat. Zametki YaGU. 1998; 5 (2): 118-134.

3. Khludnev AM, Kovtunenko VA. Analysis of cracks in solids. Southampton-Boston: WIT Press, 2000.

4. Hoffmann H-K, Khludnev AM. Fictitious domain method for the Signorini problem in a linear elasticity. Adv. Math. Sci. Appl. 2004; 14(2): 465-481. 
5. Khludnev AM. Elasticity problems in nonsmooth domains. Moscow: Fizmatlit, 2010.

6. Baiocchi C and Capelo A. Variational and quasivariational inequalities. New York, London: J Wiley and Sons, 1984.

7. Duvaut G and Lions JL. Inequalities in mechanics and physics. Berlin: Springer-Verlag, 1976.

8. Kinderlehrer D and Stampacchia G. An introduction to variational inequalities and their applications. Philadelphia: SIAM, 2000.

9. Kravchuk AS. Variational and quasivariational inequalities in mechanics. Moscow: MGAPI, 1997.

10. Lions JL. Quelques méthodes de résolution des problèmes aux limites non linéaires. Paris: Dunod Gauthier-Villars, 1969.

11. Washizu K. Variational methods in elasticity and plasticity. Oxford and New York: Pergamon Press, 1982.

12. Khludnev AM, Leugering G. On elastic bodies with thin rigid inclusions and cracks. Math. Meth. Appl. Sci. 2010; 33 (16): 1955-1967.

13. Khludnev AM. On bending an elastic plate with a delaminated thin rigid inclusion. J. Appl. Industr. Math. 2011; 5 (4): 582-594.

14. Khludnev AM. Thin rigid inclusions with delaminations in elastic plates. Eur. J. Mech. Solids. 2012; 32: $69-75$.

15. Lazarev NP. An equilibrium problem for the Timoshenko-type plate containing a crack on the boundary of a rigid inclusion. J. Siberian Federal Univ. (Mathematics and Physics) 2013; 6 (1): 53-62.

16. Neustroeva NV. A rigid inclusion in the contact problem for elastic plates. J. Appl. Industr. Math. 2010; 4 (4): 526-538.

17. Popova TS. A rigid inclusion in the problem for a viscoelastic body with a crack. Mat. Zametki YaGU. 2013; 20 (1): 87-106.

18. Fichera G. Existence theorems in elasticity. Berlin: Springer-Verlag, 1972.

19. Evans LC, Gariepi RF. Measure theory and fine properties of functions. Boca Raton, Florida: CRC Press, 1992. 